\title{
THE BELTRAMI EQUATIONS IN THREE DIMENSIONS*
}

BY

\section{E. R. HEDRICK AND LOUIS INGOLD}

1. Introduction. The equations known as Beltrami's equations may be regarded as a generalization of the Cauchy-Riemann equations. The Cauchy-Riemann equations apply to functions of a complex variable in a plane while the Beltrami equations apply to functions of a complex variable in an arbitrary surface in three dimensions referred to any pair of curvilinear coördinates.

These equations may be obtained by transforming the Cauchy-Riemann equations to curvilinear coördinates: thus suppose $(u, v)$ is a function (not necessarily analytic) of $(x, y)$. Associated with this function are quantities $E, F, G$ defined as in differential geometry:

$$
E=u_{x}^{2}+v_{x}^{2}, \quad F=u_{x} u_{y}+v_{x} v_{y}, \quad G=u_{y}^{2}+v_{y}^{2} .
$$

Thus the conditions that another function $(U, V)$ of $(x, y)$ be an analytic function of $(u, v)$ are easily found to be

$$
V_{x}=\frac{1}{\sqrt{E G-F^{2}}}\left|\begin{array}{l}
U_{x} E \\
U_{y} F
\end{array}\right|, \quad V_{y}=\frac{1}{\sqrt{E G-F^{2}}}\left|\begin{array}{c}
U_{x} F \\
U_{y} G
\end{array}\right|
$$

While these have the same form as Beltrami's equations the proof does not apply to surfaces in general, since $E, F$, and $G$ are here the coefficients of a quadratic form $E d x^{2}+2 F d x d y+G d y^{2}$ of curvature zero.

This method applied to functions in three dimensions leads to a generalization of Beltrami's equations, but here again the result would only be established for spaces of curvature zero.

The usual method for arbitrary two-dimensional surfaces makes use of the imaginary factorization of the quadratic form $E d x^{2}+2 F d x d y+G d y^{2}$. Obviously this method does not admit of generalization to higher dimensions.

In order, then, to find the analogues of Beltrami's equations for arbitrary curved spaces of three dimensions (or higher) some new method must be devised although the form of these generalizations may possibly be suggested by the first of the methods mentioned above.

\footnotetext{
* Presented to the Society, Southwestern Section, December 1, 1923.
} 
The case of space of zero curvature is of sufficient importance to deserve separate treatment. It is therefore first considered in this paper and by the method just indicated for the plane.

The case of curved spaces is then investigated and it is found that a different property of the transformations in question leads to equations of precisely the same form for this case. These equations are then used to obtain a classification of three-dimensional functions just as the Beltrami equations in the plane have been used to classify general functions of a complex variable.*

2. Extension to ordinary space. If the discussion is limited to ordinary space the generalization desired may be obtained for three dimensions, as well as for two, by combining an analytic and a nonanalytic function. Let $(u, v, w)$ be any function of $(x, y, z)$ and $(U, V, W)$ be an analytic functiont of $(u, v, w)$. Let us write

$$
\begin{aligned}
& x=f(u, v, w), \\
& y=\varphi(u, v, w), \\
& z=\psi(u, v, w) .
\end{aligned}
$$

Regarding $x, y, z$ as the independent variables and taking derivatives of both sides of each equation with respect to $x, y$, and $z$, we find

$$
\begin{array}{lll}
1=x_{u} u_{x}+x_{v} v_{x}+x_{w} w_{x}, & 0=x_{u} u_{y}+x_{v} v_{y}+x_{w} w_{y}, & 0=x_{u} u_{z}+x_{v} v_{z}+x_{w} w_{z}, \\
0=y_{u} u_{x}+y_{v} v_{x}+y_{w} w_{x}, & 1=y_{u} u_{y}+y_{v} v_{y}+y_{w} w_{y}, & 0=y_{u} u_{z}+y_{v} v_{z}+y_{w} w_{z}, \\
0=z_{u} u_{x}+z_{v} v_{x}+z_{w} w_{x}, & 0=z_{u} u_{y}+z_{v} v_{y}+z_{w} w_{y}, & 1=z_{u} u_{z}+z_{v} v_{z}+z_{w} w_{z} .
\end{array}
$$

Solving we obtain

$$
\begin{aligned}
& u_{x}=J\left|\begin{array}{ll}
y_{v} & y_{w} \\
z_{v} & z_{w}
\end{array}\right|, \quad u_{y}=J\left|\begin{array}{ll}
z_{v} & z_{w} \\
x_{v} & x_{w}
\end{array}\right|, \quad u_{z}=J\left|\begin{array}{ll}
x_{v} & x_{w} \\
y_{v} & y_{w}
\end{array}\right|, \\
& v_{x}=J\left|\begin{array}{ll}
y_{w} & y_{u} \\
z_{w} & z_{u}
\end{array}\right|, \quad v_{y}=J\left|\begin{array}{ll}
z_{w} & z_{u} \\
x_{w} & x_{u}
\end{array}\right|, \quad v_{z}=J\left|\begin{array}{ll}
x_{w} & x_{u} \\
y_{w} & y_{u}
\end{array}\right|, \\
& w_{x}=J\left|\begin{array}{ll}
y_{u} & y_{v} \\
z_{u} & z_{v}
\end{array}\right|, \quad w_{y}=J\left|\begin{array}{ll}
z_{u} & z_{v} \\
x_{u} & x_{v}
\end{array}\right|, \quad w_{z}=J\left|\begin{array}{ll}
x_{u} & x_{v} \\
y_{u} & y_{v}
\end{array}\right|,
\end{aligned}
$$

where $J$ is the jacobian of $(u, v, w)$ with respect to $(x, y, z)$. Now because $(U, V, W)$ is an analytic function of $(u, v, w)$, we have

* See Hedrick, Ingold, and Westfall, Theory of non-analytic functions of a complex variable, Journal de Mathématiques, ser. 9, vol. 2 (1923).

$\dagger$ See a paper by the authors, pp. 551-555 of the present number of these Transactions. 


$$
U_{u}=\frac{1}{\sqrt{E}}\left|\begin{array}{cc}
V_{x} & V_{v} \\
W_{v} & W_{w}
\end{array}\right|
$$

and so on; and remembering that $u, v, w$ are functions of $x, y, z$ we have

$$
\begin{aligned}
& U_{x} x_{\imath}+U_{y} y_{u}+U_{z} z_{u}=\frac{1}{\sqrt{E}}\left\{\left[x_{v} y_{w}\right]\left[V_{x} W_{y}\right]+\left[y_{v} z_{w}\right]\left[V_{y} W_{z}\right]+\left[z_{v} x_{v}\right]\left[V_{z} W_{x}\right]\right\}, \\
& U_{x} x_{v}+U_{y} y_{v}+U_{z} z_{v}=\frac{1}{\sqrt{E}}\left\{\left[x_{w} y_{u}\right]\left[V_{x} W_{y}\right]+\left[y_{w} z_{u}\right]\left[V_{y} W_{z}\right]+\left[z_{w} x_{u}\right]\left[V_{z} W_{x}\right]\right\}, \\
& U_{x} x_{w}+U_{y} y_{w}+U_{z} z_{w}=\frac{1}{\sqrt{E}}\left\{\left[x_{u} y_{v}\right]\left[V_{x} W_{y}\right]+\left[y_{u} z_{v}\right]\left[V_{y} W_{z}\right]+\left[z_{u} x_{v}\right]\left[V_{z} W_{x}\right]\right\},
\end{aligned}
$$

where the small brackets on the right represent second order determinants, according to the usual notation.

These equations may be solved for $U_{x}$ by multiplying in order by $u_{x}, v_{x}, w_{x}$ and adding. The result on the right can be reduced by using the values for $u_{x}, v_{x}$, etc. found above. Similarly $U_{y}$ and $U_{z}$ can be found; and also

$$
V_{x}, V_{y}, V_{z} \text {, and } W_{x}, W_{y}, W_{z} .
$$

The final results are given below; the $E_{i j}$ are the coefficients of the differential form connecting $(u, v, w)$ with $(x, y, z)$ and $E$ is the coefficient of the differential form connecting $(U, V, W)$ with $(u, v, w):^{*}$

(2)

$$
\begin{gathered}
U_{x}=\frac{1}{J \sqrt{E}}\left|\begin{array}{ccc}
E_{11} & E_{12} & E_{13} \\
V_{x} & V_{y} & V_{z} \\
W_{x} & W_{y} & W_{z}
\end{array}\right|, \quad U_{y}=\frac{1}{J \sqrt{\bar{E}}}\left|\begin{array}{ccc}
E_{21} & E_{22} & E_{23} \\
V_{x} & V_{y} & V_{z} \\
W_{x} & W_{y} & W_{z}
\end{array}\right|, \\
U_{z}=\frac{1}{J \sqrt{\bar{E}}}\left|\begin{array}{ccc}
E_{31} & E_{32} & E_{33} \\
V_{x} & V_{y} & V_{z} \\
W_{x} & W_{y} & W_{z}
\end{array}\right|,
\end{gathered}
$$

$$
\begin{gathered}
V_{x}=\frac{1}{J \sqrt{E}}\left|\begin{array}{lll}
U_{x} & U_{y} & U_{z} \\
E_{11} & E_{12} & E_{13} \\
W_{x} & W_{y} & W_{z}
\end{array}\right|, \quad V_{y}=\frac{1}{J \sqrt{E}}\left|\begin{array}{lll}
U_{x} & U_{y} & U_{z} \\
E_{21} & E_{28} & E_{23} \\
W_{x} & W_{y} & W_{z}
\end{array}\right|, \\
V_{z}=\frac{1}{J \sqrt{E}}\left|\begin{array}{lll}
U_{x} & U_{y} & U_{z} \\
E_{31} & E_{32} & E_{33} \\
W_{x} & W_{y} & W_{z}
\end{array}\right|,
\end{gathered}
$$

* In this case only one of the fundamental quantities need be used, since, in the analytic case, $E_{11}=E_{23}=E_{33}=E$ and $E_{i j}=0$ if $i \neq j$. See papers by the authors, loc. cit. 


$$
\begin{gathered}
W_{x}=\frac{1}{J \sqrt{\bar{E}}}\left|\begin{array}{lll}
U_{x} & U_{y} & U_{z} \\
V_{x} & V_{y} & V_{z} \\
E_{11} & E_{18} & E_{13}
\end{array}\right|, \quad W_{y}=\frac{1}{J \sqrt{E}}\left|\begin{array}{lll}
U_{x} & U_{y} & U_{z} \\
V_{x} & V_{y} & V_{z} \\
E_{81} & E_{22} & E_{23}
\end{array}\right|, \\
W_{z}=\frac{1}{J \sqrt{E}}\left|\begin{array}{lll}
U_{x} & U_{y} & U_{z} \\
V_{x} & V_{y} & V_{z} \\
E_{31} & E_{32} & E_{33}
\end{array}\right| .
\end{gathered}
$$

These equations may also be written in the form

(3) $\left|\begin{array}{ll}U_{y} & V_{y} \\ U_{z} & V_{z}\end{array}\right|=\frac{V \bar{E}}{J}\left|\begin{array}{lll}W_{x} & E_{12} & E_{13} \\ W_{y} & E_{22} & E_{23} \\ W_{z} & E_{32} & E_{33}\end{array}\right|,\left|\begin{array}{ll}U_{z} & V_{z} \\ U_{x} & V_{x}\end{array}\right|=\frac{V \bar{E}}{J}\left|\begin{array}{lll}E_{11} & W_{x} & E_{13} \\ E_{12} & W_{y} & E_{23} \\ E_{13} & W_{z} & E_{33}\end{array}\right|$, etc.

3. Properties of Beltrami's equations. Writing out the values for $U_{x}, V_{x}, W_{x}$, we have*

$$
\begin{aligned}
& U_{x}=\frac{1}{J \sqrt{E}}\left\{E_{11}\left|\begin{array}{cc}
V_{y} & V_{z} \\
W_{y} & W_{z}
\end{array}\right|+E_{12}\left|\begin{array}{cc}
V_{z} & V_{x} \\
W_{z} & W_{x}
\end{array}\right|+E_{13}\left|\begin{array}{cc}
V_{x} & V_{y} \\
W_{x} & W_{y}
\end{array}\right|\right\}, \\
& V_{x}=\frac{1}{J V \bar{E}}\left\{E_{11}\left|\begin{array}{l}
W_{y} W_{z} \\
U_{y} U_{z}
\end{array}\right|+E_{12}\left|\begin{array}{l}
W_{z} W_{x} \\
U_{z} U_{x}
\end{array}\right|+E_{13}\left|\begin{array}{c}
W_{x} W_{y} \\
U_{x} U_{y}
\end{array}\right|\right\} \text {, } \\
& W_{x}=\frac{1}{J V \bar{E}}\left\{E_{11}\left|\begin{array}{ll}
U_{y} & U_{z} \\
V_{y} & V_{z}
\end{array}\right|+E_{12}\left|\begin{array}{ll}
U_{z} & U_{x} \\
V_{z} & V_{x}
\end{array}\right|+E_{13}\left|\begin{array}{cc}
U_{x} & U_{y} \\
V_{x} & V_{y}
\end{array}\right|\right\} \text {. }
\end{aligned}
$$

Multiplying the first by $U_{x}$, the second by $V_{x}$, and the third by $W_{x}$ and adding, we find

$$
U_{x}^{2}+V_{x}^{2}+W_{x}^{2}=\frac{J^{\prime}}{J \sqrt{E}} E_{11}
$$

where $J^{\prime}$ is the jacobian of $(U, V, W)$ with respect to $(x, y, z)$.

Similarly

$$
\begin{aligned}
U_{x} U_{y}+V_{x} V_{y}+W_{x} W_{y} & =\frac{J^{\prime}}{J V \bar{E}} E_{12} \\
U_{x} U_{z}+V_{x} V_{z}+W_{x} W_{z} & =\frac{J^{\prime}}{J V \bar{E}} E_{13}
\end{aligned}
$$

From the expanded forms for $U_{y}, V_{y}, W_{y}$, and $U_{z}, V_{z} W_{z}$, analogous formulas can be derived.

Thus if $(U, V, W)$ is an analytic function of $(u, v, w)$, the fundamental quantities of the function $(U, V, W)=\Phi(x, y, z)$ are proportional to the

* The special analytic case, in which $E_{11}=E_{23}=E_{28}=E$ and $E_{i j}=0$ if $i \neq j$ obviously gives the Cauchy-Riemann equations of the earlier paper. 
fundamental quantities of $(u, v, w)=F(x, y, z)$. This is a case of the extension of the Beltrami theorem that two "functions on the same surface" are analytic functions of each other. The general case is given in $\S 5$. It is clear also that two different analytic functions of $(u, v, w)$ have fundamental quantities that are proportional when both are regarded as functions of $(x, y, z)$.

4. Extension to curved spaces. Consider a curved surface with the fundamental quantities $E, F, G$; let $t_{1}$ and $t_{2}$ be tangent vectors to the parameter curves such that $t_{1} t_{2}=F, t_{1} t_{1}=E, t_{2} t_{2}=G$.

If $u(x, y)=c$ determines a family of curves on the surface, the vector

$$
\frac{\partial u}{\partial x} t_{2}-\frac{\partial u}{\partial y} t_{1}
$$

is at each point tangent to $u=c$. If $v=k$ is another family of curves on the surface, the vector

$$
\left(F \frac{\partial v}{\partial y}-G \frac{\partial v}{\partial x}\right) t_{1}+\left(F \frac{\partial v}{\partial x}-E \frac{\partial v}{\partial y}\right) t_{2}
$$

is at each point orthogonal to $v=k$. The condition that $u=c$ be the orthogonal trajectories of $v=k$ is

$$
F \frac{\partial v}{\partial y}-G \frac{\partial v}{\partial x}=-h \frac{\partial u}{\partial y}, \quad F \frac{\partial v}{\partial x}-E \frac{\partial v}{\partial y}=h \frac{\partial u}{\partial x}
$$

where $h$ is a scalar factor.

If, in addition, the two invariants* $\Delta_{1} u, \Delta_{1} v$ are to be equal it is found that $h$ must be equal to $\sqrt{E G-F^{\mathbf{2}}}$. Thus the necessary and sufficient conditions that $u=c$ and $v=k$ are the orthogonal trajectories of each other and that $\Delta_{1} u=\Delta_{1} v$ are

$$
\frac{\partial u}{\partial y}=\frac{1}{\sqrt{E G-F^{\mathbf{2}}}}\left|\begin{array}{ll}
F & \frac{\partial v}{\partial x} \\
G & \frac{\partial v}{\partial y}
\end{array}\right|, \quad \frac{\partial u}{\partial x}=\frac{1}{\sqrt{E G-F^{\mathbf{2}}}}\left|\begin{array}{ll}
E & \frac{\partial v}{\partial x} \\
F & \frac{\partial v}{\partial y}
\end{array}\right|,
$$

and these are Beltrami's equations. We now propose a similar problem in three dimensions: Consider a curved, three-dimensional space with the

$* \Delta_{1} \Phi$ is the differential parameter

$$
\left[E\left(\frac{\partial \Phi}{\partial y}\right)^{2}-2 F \frac{\partial \Phi}{\partial x} \frac{\partial \Phi}{\partial y}+G\left(\frac{\partial \Phi}{\partial x}\right)^{2}\right] \div(E G-F) .
$$


fundamental quantities $E_{i j}$; also let $t_{1}, t_{2}, t_{3}$ be three vectors tangent to the parameter cur;es such that $t_{i} t_{j}=E_{i j}$. The tangent vector to the curve of intersection of two surfaces $U=c, V=k$ can be written in the form

$$
\left|\begin{array}{ll}
U_{y} & V_{y} \\
U_{z} & V_{z}
\end{array}\right| t_{1}+\left|\begin{array}{ll}
U_{z} & V_{z} \\
U_{x} & V_{x}
\end{array}\right| t_{2}+\left|\begin{array}{ll}
U_{x} & V_{x} \\
U_{y} & V_{y}
\end{array}\right| t_{3} .
$$

The normal vector to a surface $W=h$ has the form

$$
\left|\begin{array}{lll}
W_{x} & E_{12} & E_{13} \\
W_{y} & E_{22} & E_{23} \\
W_{z} & E_{32} & E_{33}
\end{array}\right| t_{1}+\left|\begin{array}{ccc}
E_{11} & W_{x} & E_{13} \\
E_{12} & W_{y} & E_{23} \\
E_{13} & W_{z} & E_{33}
\end{array}\right| t_{2}+\left|\begin{array}{ccc}
E_{11} & E_{21} & W_{x} \\
E_{12} & E_{22} & W_{y} \\
E_{13} & E_{23} & W_{z}
\end{array}\right| t_{3} .
$$

Hence the necessary and sufficient condition that the surfaces $W=h$ be the orthogonal trajectories of the curves $U=c, V=k$ are given by the equations

$$
\left|\begin{array}{c}
W_{x} E_{12} E_{13} \\
W_{y} E_{28} E_{23} \\
W_{z} E_{32} E_{33}
\end{array}\right|=p\left|\begin{array}{c}
U_{y} V_{y} \\
U_{z} V_{z}
\end{array}\right|,\left|\begin{array}{l}
E_{11} W_{x} E_{13} \\
E_{12} W_{y} E_{23} \\
E_{13} W_{z} E_{33}
\end{array}\right|=p\left|\begin{array}{c}
U_{z} V_{z} \\
U_{x} V_{x}
\end{array}\right|,\left|\begin{array}{c}
E_{11} E_{21} W_{x} \\
E_{12} E_{28} W_{y} \\
E_{13} E_{23} W_{z}
\end{array}\right|=p\left|\begin{array}{c}
U_{x} V_{x} \\
U_{y} V_{y}
\end{array}\right|,
$$

where $p$ is a factor of proportionality.

These three equations may be solved for $W_{x}, W_{y}, W_{z}$. The resulting values are

$$
W_{x}=P\left|\begin{array}{lll}
U_{x} & U_{y} & U_{z} \\
V_{x} & V_{y} & V_{z} \\
E_{11} & E_{12} & E_{13}
\end{array}\right|, \quad W_{y}=P\left|\begin{array}{ccc}
U_{x} & U_{y} & U_{z} \\
V_{x} & V_{y} & V_{z} \\
E_{21} & E_{22} & E_{23}
\end{array}\right|, \quad W_{z}=P\left|\begin{array}{ccc}
U_{x} & U_{y} & U_{z} \\
V_{x} & V_{y} & V_{z} \\
E_{31} & E_{32} & E_{33}
\end{array}\right|,
$$

where $P$ is a new factor of proportionality.

If it is required that $U=c$ be the orthogonal trajectories of the curves $V=k, W=h$, and that $V=k$ be the orthogonal trajectories of $W=h$, $U=c$, we obtain

$$
\begin{aligned}
& U_{x}=P\left|\begin{array}{lll}
E_{11} & E_{12} & E_{13} \\
V_{x} & V_{y} & V_{z} \\
W_{x} & W_{y} & W_{z}
\end{array}\right|, \quad U_{y}=P\left|\begin{array}{ccc}
E_{21} & E_{22} & E_{23} \\
\nabla_{x} & V_{y} & V_{z} \\
W_{x} & W_{y} & W_{z}
\end{array}\right|, \quad U_{z}=P\left|\begin{array}{ccc}
E_{31} & E_{38} & E_{\mathrm{s} 3} \\
V_{x} & V_{y} & V_{z} \\
W_{x} & W_{y} & W_{z}
\end{array}\right|, \\
& V_{x}=P\left|\begin{array}{lll}
U_{x} & U_{y} & U_{z} \\
E_{11} & E_{12} & E_{13} \\
W_{x} & W_{y} & W_{z}
\end{array}\right|, \quad W_{y}=P\left|\begin{array}{ccc}
U_{x} & U_{y} & U_{z} \\
E_{21} & E_{23} & E_{23} \\
W_{x} & W_{y} & W_{z}
\end{array}\right|, \quad W_{z}=P\left|\begin{array}{ccc}
U_{x} & U_{y} & U_{z} \\
E_{31} & E_{32} & E_{33} \\
W_{x} & W_{y} & W_{z}
\end{array}\right| .
\end{aligned}
$$

These are the Beltrami equations for a curved space. 
5. Functions in curved space. It is easy to show as in $\S 3$ that

$$
\begin{array}{lll}
\sum U_{x}^{2}=Q E_{11}, & \sum U_{y}^{2}=Q E_{22}, & \sum U_{z}^{2}=Q E_{33}, \\
\sum U_{x} U_{y}=Q E_{12}, & \sum U_{y} U_{z}=Q E_{93}, & \sum U_{z} U_{x}=Q E_{31},
\end{array}
$$

provided that $U, V$, and $W$ satisfy Beltrami's equations, where $Q$ is a factor of proportionality whose value can be found readily. Similar results hold for a function $U^{\prime}, V^{\prime}, W^{\prime}$ except that the proportionality factor $Q^{\prime}$ may be different from $Q$; consequently if $(U, V, W)$ and $\left(U^{\prime}, V^{\prime}, W^{\prime}\right)$ are two functions satisfying Beltrami's equations, we have

$$
\begin{array}{ll}
\sum U_{x}^{2}=R \sum U_{x}^{\prime 2}, & \sum U_{x} U_{y}=R \sum U_{x}^{\prime} U_{y}^{\prime}, \\
\sum U_{y}^{2}=R \sum U_{y}^{\prime 2}, & \sum U_{y} U_{z}=R \sum U_{y}^{\prime} U_{z}^{\prime}, \\
\sum U_{z}^{2}=R \sum U_{z}^{\prime 2}, & \sum U_{z} U_{x}=R \sum U_{z}^{\prime} U_{x}^{\prime} .
\end{array}
$$

Thus $(U, V, W)$ is an analytic function of $\left(U^{\prime}, V^{\prime}, W^{\prime}\right)$, by $\S 4$ of the preceding paper (p. 553). These equations constitute the essential generalization of the Beltrami theorem mentioned in $\S 3$.

6. Non-analytic functions. Let $u, v, w$ be any function $F(x, y, z)$ in ordinary space, and denote the fundamental quantities of this function by $E_{i j}$. By $\S 3$ the fundamental quantities of $(U, V, W)=\Phi(x, y, z)$ are proportional to $E_{i j}$ provided $(U, V, W)$ is an analytic function of $(u, v, w)$.

The converse of this is also true. If the fundamental quantities of $\Phi(x, y, z)$ are proportional to the fundamental quantities of the function $F(x, y, z)$, then $\Phi$ is an analytic function of $F$. Thus the ratios of the fundamental quantities $E_{i j}$ determine a class of functions which are analytic functions of each other.*

With suitable conditions as to continuity and differentiability the following statements are easily proved:

Every function belongs to a definite class in a given region.

No function belongs to two different classes in the same region.

The totality of analytic functions in a given region constitutes a separate class. There exist functions belonging to every class in a given region.

* This is the extension to three dimensions of the method of classification of functions of a complex variable given in the paper Non-analytic functions, etc., loc. cit.

The University of California, | The University of Missouri, Southern Branch; 